Cahiers $d u$ MONDE RUSSE

\section{Cahiers du monde russe}

Russie - Empire russe - Union soviétique et États indépendants

$51 / 4 \mid 2010$

Sciences humaines et sociales en Russie à l'Âge d'argent

\title{
Frances L. Bernstein, Christopher Burton, Dan Healey, eds., Soviet Medicine
}

\section{Grégory Dufaud}

\section{OpenEdition}

\section{Journals}

Édition électronique

URL : https://journals.openedition.org/monderusse/7385

DOI : 10.4000/monderusse. 7385

ISSN : $1777-5388$

Éditeur

Éditions de l'EHESS

\section{Édition imprimée}

Date de publication : 25 novembre 2010

Pagination : 706-708

ISBN : 978-2-7132-2316-7

ISSN : $1252-6576$

Référence électronique

Grégory Dufaud, «Frances L. Bernstein, Christopher Burton, Dan Healey, eds., Soviet Medicine », Cahiers du monde russe [En ligne], 51/4 | 2010, mis en ligne le 28 novembre 2019, consulté le 04 septembre 2022. URL : http://journals.openedition.org/monderusse/7385 ; DOI : https://doi.org/ 10.4000/monderusse.7385

Ce document a été généré automatiquement le 4 septembre 2022.

Tous droits réservés 


\title{
Frances L. Bernstein, Christopher Burton, Dan Healey, eds., Soviet Medicine
}

\author{
Grégory Dufaud
}

\section{RÉFÉRENCE}

Frances L. Bernstein, Christopher Burton, Dan Healey, eds., Soviet Medicine. Culture,

Practice and Science. Dekalb : Northern Illinois University Press, 2010, 294 p.

1 Quelques mois après la révolution, les bolcheviks entreprirent de construire un système de santé publique censé concourir à la « reformation» des individus et à l'avènement d'un homo sovieticus actif et en bonne santé. Le recueil dirigé par Frances Bernstein, Christopher Burton et Dan Healey réunit douze articles, ordonnés selon une logique chronologique, qui interrogent le projet médical soviétique et renseignent sur les pratiques médicales pour l'ensemble de la période : les six premiers concernent l'entredeux-guerres et les six autres l'après-guerre. Les textes sont mis en perspective par une introduction qui dépeint les enjeux du livre, les articulations majeures de l'histoire médicale soviétique et les principaux jalons de l'historiographie de la santé.

2 Ce recueil présente un intérêt majeur, tant du point de vue empirique qu'historiographique. La santé y apparait comme un domaine fécond d'investigation grâce à des contributions qui éclairent le fonctionnement de l'État soviétique non seulement dans ce qu'il pouvait représenter de meilleur mais aussi dans ses aspects les plus indignes. Cinq grands thèmes peuvent être dégagés.

3 Le premier a trait à l'organisation du champ médical. Irina Sirotkina (ch. I) s'intéresse au processus d'institutionnalisation de la psychiatrie au lendemain de la révolution. Celuici marqua, selon l'auteur, l'échec du projet libéral porté par les psychiatres depuis 1905 en ce sens qu'il conduisit à un contrôle accru de l'État sur la profession. Frances Bernstein (ch. IV) montre, à travers la question du secret médical en vénérologie, que le 
rapport des médecins à l'État fut néanmoins l'objet de négociations au cours des années 1920, l'enjeu étant la relation au patient. Malgré le souhait du régime d'abolir le secret médical en tant que « relique » du passé tsariste, celui-ci continua à être appliqué. Les médecins devaient toutefois obliger les malades à se soigner et signaler les malades qui infectaient en connaissance de cause d'autres personnes. La tension entre répression et surveillance fut certainement à l'origine de la réforme de l'avortement dans l'aprèsguerre. Comme Mie Nakachi le souligne (ch.IX), nombre d'obstétriciens et de gynécologues ignoraient délibérément la législation de 1936 et ne dénonçaient pas les avortements qu'ils n'avaient pas pratiqués eux-mêmes. Ils plaçaient ainsi l'intérêt de la patiente au-dessus de celui de l'État. Confrontées à un hiatus toujours plus important entre la loi et son application, les autorités élargirent les critères légaux de l'avortement en 1951, puis le légalisèrent en 1955. Dans une enquête sur la pédiatrie à la fin de la période soviétique, Catriona Kelly (ch. XII) révèle l'attachement que les Russes portaient alors à leur médecin et au personnel médical dont les interviewés soulignent le dévouement. Tout simplement, ils nourrissaient une grande confiance à leur endroit.

Dans les chapitres consacrés à la lutte contre la peste sur la basse Volga (II) et à la figure de l'Allemand Max Kuczynski (III), la médecine est placée en terrain colonial. Dmitry Mikhel fait bien voir que les mesures prises contre la peste débordèrent le domaine sanitaire pour devenir un outil de contrôle et d'acculturation des populations dites " arriérées". La transformation de leur mode de vie ancestral devait leur assurer une meilleure santé et donc leur permettre de mieux résister aux infections. Max Kuczynski, étudié par Susan Gross Solomon, est un jeune pathologiste qui, après avoir séjourné plusieurs mois en Sibérie en 1923-1924 pour y observer le rôle de l'environnement sur les maladies, développa la notion d'« ethnopathologie ». Contrairement à la majorité de ses collègues russes, il jugeait que les nomades savaient mieux que quiconque ce qui leur convenait, et se montrait des plus sceptiques à l'égard de la mission civilisatrice soviétique.

5 Michael Z. David, étudiant la vaccination contre la tuberculose entre 1925 et 1941 (ch. VI), présente la mise en œuvre d'un programme de santé publique. Très tôt, dès le début des années 1920, le Commissariat à la santé (Narkomzdrav) développa un vaste réseau de dispensaires pour traiter la tuberculose. Le recours au BCG - résultat d'une coopération scientifique avec l'Institut Pasteur - devait venir compléter le dispositif de prévention. La vaccination n'en était pas moins soumise à l'accord des parents. Promue par les activistes du Narkomzdrav qui obtinrent le budget nécessaire, elle dut toutefois son succès à l'implication des médecins en province. Il reste que la carte des vaccinés avant 1946 correspondait à celle des grands centres industriels.

6 Les médecins furent aussi appelés à jouer le rôle d'expert. Dan Healey (ch. V) aborde la notion de « maturité sexuelle », établie pour remplacer celle d'âge légal qui imposait un même standard à des individus physiologiquement différents. Dans les faits, cette notion souleva des complications qui conduisirent les médecins à prendre en compte le développement physique et les caractéristiques sexuelles. Chez la femme, la maturité sexuelle fut souvent réduite à la fonction reproductive. Marina Sorokina (ch. VII) se penche sur la commission dite «Burdenko » (du nom du médecin l'ayant dirigée) qui fut missionnée à Katyń pour confirmer la version soviétique du massacre. Il semblerait que la falsification fut moins le produit d'un ordre venu d'en haut que d'un dialogue entre autorités et praticiens afin de parvenir aux conclusions adéquates : « the medical experts were not simply "programmed" to falsify the results of the exhumations in advance». 
Veniamin Zima (ch. VIII) montre comment la famine de 1946-1947, et les épidémies qu'elle suscita, stimulèrent la constitution d'un savoir substantiel par le biais de recherches approfondies. Celui-ci fut toutefois peu mis en œuvre par les autorités qui se contentèrent de promouvoir l'amélioration des conditions sanitaires. L'auteur pointe la collusion des experts et des responsables politiques pour masquer la réalité de la catastrophe.

7 Le dernier thème concerne la situation sanitaire de l'Union soviétique dans l'aprèsguerre, à un moment où le pays connaît un nouveau boom industriel. Les infrastructures urbaines soviétiques présentaient un retard considérable par rapport à l'Europe, alors même que des technologies modernes étaient disponibles. Donald Filtzer s'intéresse spécifiquement à l'eau (ch. X). Il souligne la médiocrité de l'approvisionnement tant du point de vue qualitatif que quantitatif, l'ampleur de la pollution industrielle et la négligence des responsables d'usines. Tout simplement, le régime sacrifiait la santé de sa population et la qualité de son environnement au nom de ses ambitions économiques. Sous Hruščev, une nouvelle impulsion fut donnée à l'« hygiène communautaire", discipline médicale qui s'intéressait aux facteurs externes de la santé humaine. Mais, comme le montre le débat sur les toxines industrielles étudié par Christopher Burton (ch. XI), toute critique trop radicale formulée à l'encontre du modèle de développement était rejetée à la fois par les collègues et les pouvoirs publics.

En définitive, ce recueil invite à repenser les relations de l'État soviétique à ses experts et à sa population en dépassant les oppositions tranchées, grâce à une grande attention aux situations et aux contextes. On aurait néanmoins aimé voir développer un aspect qui n'est que trop rapidement évoqué, celui de la spécificité de la pratique médicale soviétique. Cette remarque n'est nullement une critique, mais bien une interrogation suscitée par les éléments de comparaison offerts çà et là et qui donnent envie d'en savoir plus. 